\title{
Effect of fog-haze on the hydrophobicity of Silicone Rubber in winter
}

\author{
Jianhui Wang ${ }^{1,2, a}$, Yueju Zhao ${ }^{1,2, b}$,Jilin Teng ${ }^{1,2, c}$, Wenhua Miao ${ }^{1,2}$, Guogang \\ Wang $^{1,2}$, Mingjun Sun ${ }^{3}$ \\ ${ }^{1}$ Nari group corporation(state grid electric power search stitute), Beijing, 100070, China \\ ${ }^{2}$ Beijing Guodian Futong science and technology development corporation, Ltd, Bejing, 100070, \\ China \\ ${ }^{3}$ Liaoning Hongyanhe Nuclear power Co., Ltd, Dalian, 100070, China \\ aemail: 2001070010@163.com, bemail:zhaoyj@icass.ac.cn, 'email:tengjilin@sgepri.sgcc.com.cn,
}

Keywords: Silicone Rubber; Fog-haze; hydrophobicity

\begin{abstract}
Recently, fog-haze weather has become very frequent, and have a great impact on human life and production, especially for power transmission lines. In this paper, silicone rubber samples were contaminated in simulated winter fog-haze chamber for certain time. It was found that winter fog-haze environment would have a negative impact on the transfer of hydrophobicity. Combined with results of NSDD and ESDD test and hydrophobic classification, it was confirmed that pollution degree and low temperature were the main factors for the hydrophobicity and hydrophobic transfer.
\end{abstract}

\section{Introduction}

In the recent, silicone rubber materials were widely used in electric power transmission line, because of its good hydrophobicity and the transfer of the hydrophobicity, which is associated with the chemical construction of polysiloxane. The Si-O chain was surrounded by the $\mathrm{CH}_{3}$ groups, which make the interface energy very low, and surface is very hydrophobic. Even though the surface is contaminated, the low molecular weight matter and chain segment migrated out of the silicone rubber could transfer onto the dirt, that make the dirt own hydrophobicity as well. This is what the essence of the transfer of hydrophobicity[1-2].

However, long-term outdoor service could suffer aging impact from various environmental factors[3-6], such as ultraviolet radiation, rain wash, pollution accumulating and so on, which will weaken the hydrophobicity, insulating properties, mechanical strength distinctly. Xu etal[7] found that the hydrophobicity recovery speed would decline with the decline of the ambient temperature. The increase in humidity lead to more transfer time, the transfer of small molecular was suppressed by air moisture. There are others reports[8-10], which found whether pollution accumulating would made an impact on the hydrophobicity depended on the pollution nature. The effect of NSDD was apparently higher than ESDD.

In conclusion, pollution accumulating, high moisture, and low temperature would all hindered the play of transfer of hydrophobicity. While the winter hazes weather happened to cover all three factors simultaneously. So, fog-haze would make a significant effect on the safety of electric power transmission lines, and further research on this aspect would be of great scientific application value.

In this paper a series of tests were designed to uncover the influence rule of winter fog-haze, and to confirm the key factors and boundary conditions.

\section{Experimental Preparation}

Fog-haze environment was simulated by generating the main components of haze, which were then inlet into the chamber, mixed by diffusion. The final haze environment parameters were listed as follows( Table 1). 
Table1 haze environment parameters

\begin{tabular}{cc}
\hline parameters & value \\
\hline Particle concentration & $1800 \mu \mathrm{g} / \mathrm{m}^{3}$ \\
The mist conductivity & $1500 \mu \mathrm{s} / \mathrm{cm}$ \\
Relative humidity & $75 \% \sim 85 \%$ \\
Temperature & $5 \sim 10^{\circ} \mathrm{C}$
\end{tabular}

$2 \mathrm{~mm}$ thick samples made of silicone rubber were prepared, then fully dried, and cuted into saveral square plates with $5 \mathrm{~cm}$ side length for following test.

\section{Results and Discussion}

Place the plates in the fog chamber for a certain time(5days, 10days and 20days) to stain, and take out and record the surface morphology at different stage of contamination(Fig.1).

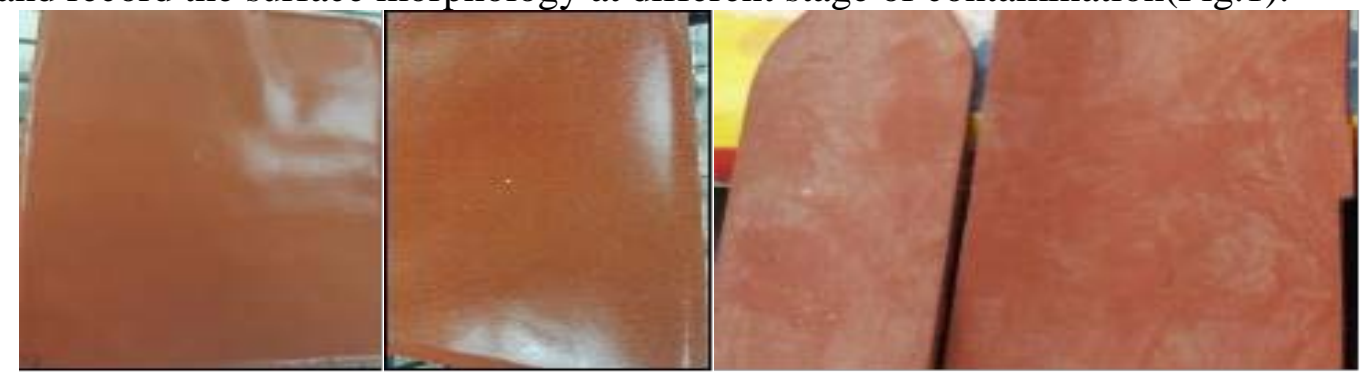

(a) Accumulated dirt before

(b) 5 days of contamination

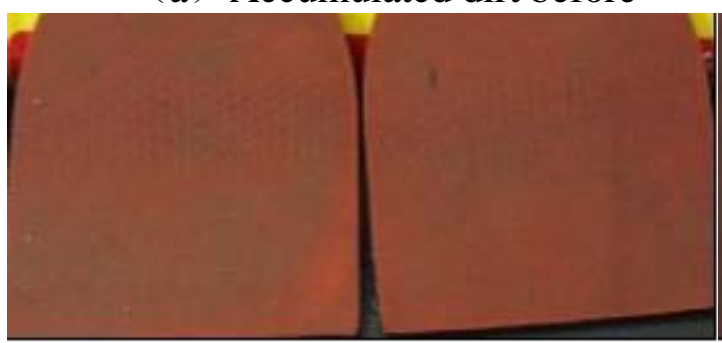

(c) 10 days of contamination

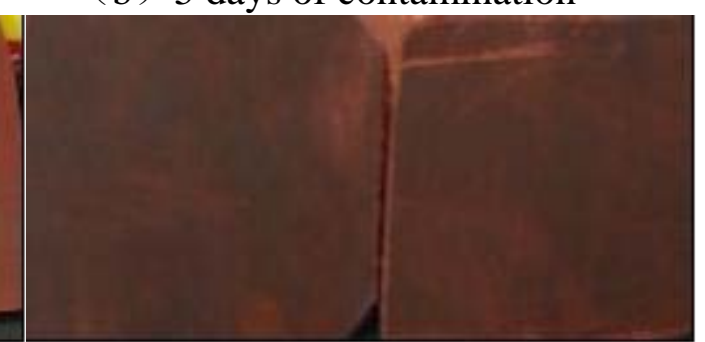

(d) 20 days of contamination

Fig.1. Surface morphology at different stage of contamination

\section{Effect of winter fog-haze on transfer of hydrophobicity.}

The samples were stayed enough long time for migration of low molecular weigh matter and segament chain. Using water spray classification to test surface hydrophobicity, the result was shown in the figure 2 .
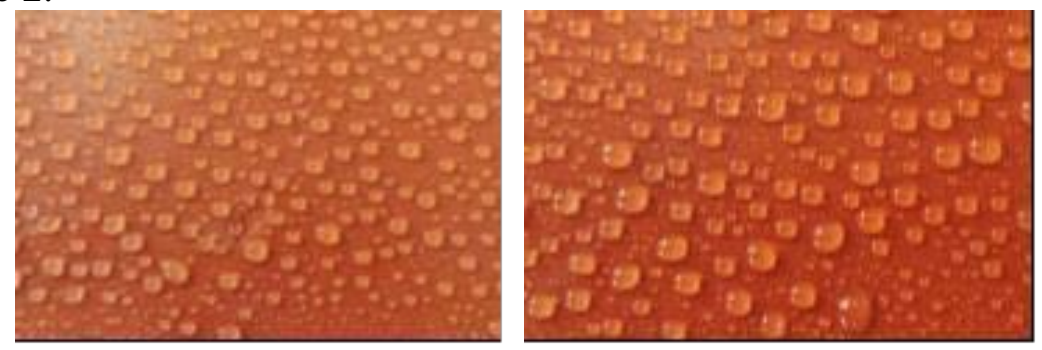

(a)clean samples

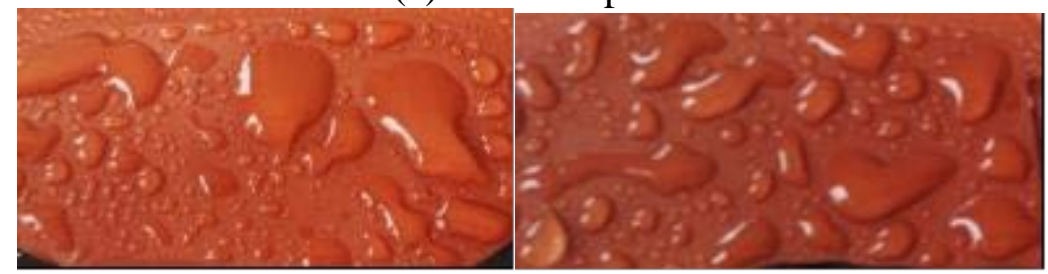

(b) 5 days of contamination 


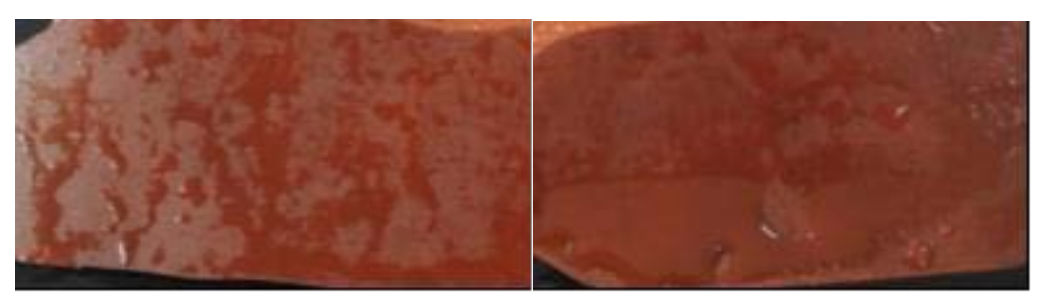

(c)10 days of contamination

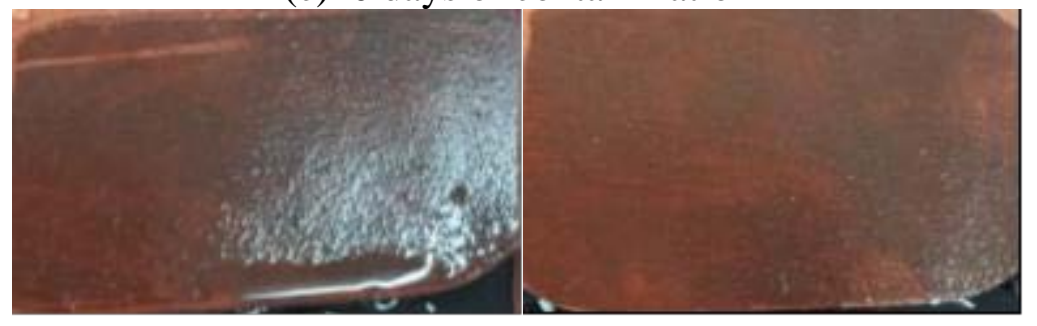

(d)20 days of contamination

Fig.2. hydrophobicity test of different fouling degree of samples

It's observed that fouling under fog-haze environment could made bad effect on the hydrophobicity, before contamination, the hydrophobicity is very good, and could be classified as HC1, however, the classification was drop to HC4 only after fouling five days, and HC5-HC6 after fouling eight days, and below the HC6 after 20 days. As seen above, accumulation fouling under haze would reduce the hydrophobicity, and hinder the migration to paly. This is related to the fog nature and fouling time length, the longer fouling time was, the greater the influence on the hydrophobicity was.

However, contact angle of polluted samples didn't decrease, they were all improved inversely (table2). For example, contact angle of contaminated 5 and 10 day's samples can reach $130^{\circ}$, that of 20 days have a little decline to $125.4^{\circ}$. Compared with clean samples, they were all enhanced.

Table 2 Test result of contact angle and hydrophobicity classification

\begin{tabular}{clllcrrrc}
\hline \multirow{5}{*}{$\begin{array}{c}\text { Clean } \\
\text { sample }\end{array}$} & sample & \multicolumn{1}{c}{$\theta 1$} & \multicolumn{1}{c}{$\theta 2$} & $\theta 3$ & $\theta 4$ & $\theta 5$ & $\theta_{\mathrm{av}}$ & HC \\
& Sample1 & 116 & 114.3 & 112.7 & 109.2 & 109.9 & 112.4 & HC1 \\
& Sample2 & 110.1 & 111.4 & 112.3 & 115.1 & 113.5 & 112.5 & HC1 \\
& Sample3 & 112.9 & 113 & 110.2 & 114.3 & 103.9 & 110.9 & HC1 \\
\hline \multirow{5}{*}{ 5days } & average & & & & & & 111.9 & HC1 \\
& Sample1 & 137.5 & 139.3 & 143.0 & 140.4 & 137.2 & 139.5 & HC3-4 \\
& Sample2 & 132.1 & 130.4 & 130.6 & 121.8 & 129.7 & 128.9 & HC4 \\
& Sample3 & 123.6 & 124.6 & 130.3 & 129.4 & 127.0 & 127.0 & HC4 \\
& average & & & & & & 131.8 & HC4 \\
\hline \multirow{5}{*}{ 10days } & Sample1 & 119.4 & 124.8 & 126.7 & 126.8 & 132.5 & 126.0 & HC5 \\
& Sample2 & 137.5 & 136.4 & 135.9 & 140.3 & 140.5 & 138.1 & HC5-HC6 \\
& Sample3 & 130.1 & 130.4 & 138.7 & 135.6 & 135.1 & 134.0 & HC5-HC6 \\
& average & & & & & & 132.7 & HC5-HC6 \\
\hline \multirow{5}{*}{ 20days } & Sample1 & 123.0 & 119.6 & 120.4 & 110.4 & 120.3 & 118.7 & Below HC6 \\
& Sample2 & 137.3 & 139.1 & 131.8 & 129.9 & 134.2 & 134.5 & Below HC6 \\
& Sample3 & 125.4 & 120.1 & 124.4 & 122.5 & 122.1 & 122.9 & HC6 \\
& average & & & & & & 125.4 & Below HC6 \\
\hline
\end{tabular}

Two methods of testing hydrophobicity seem inconsistent in result. According to the hydrophobicity classification, hydrophobic property was getting worse with the degree of accumulating pollution increased, but, from the point of contact angle test, contact angle was creasing, which mean water repellency is improved. This contradiction may be related to its micro structure of surface. It can be seen from the figure 3, for samples of 5days and 10 days contamination, dirt particles scattered with a certain distance to build a great rough surface, but for those of 20days, pollution particles heap together to form a dense layer, this surface roughness was smaller than the former. So the contact angle of former was larger than the latter. However, when 
such microstructures are subjected to external force, such as rain, they would be destroyed, so the result obtained by water pray classification was different from that by static contact angle test. The former is more closer to actual condition.

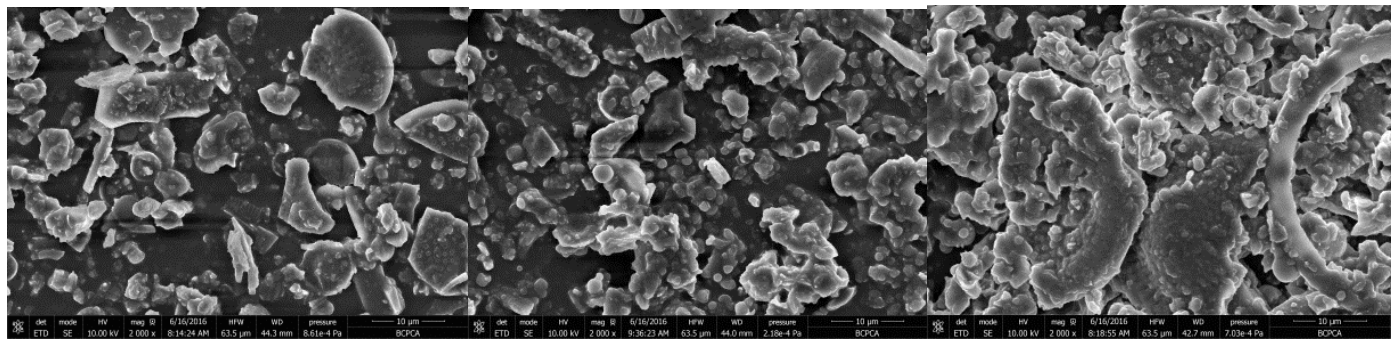

(a) 5days

(b) 10days

(c) 20days

Fig.3 SEM result of contaminated samples

We cleaned the surface of the dirt samples and tested the hydrophobicity by classification in the following experiments. It's found that the water repellency recovered to HC1-HC2. This showed that contamination was definitely an important factor.

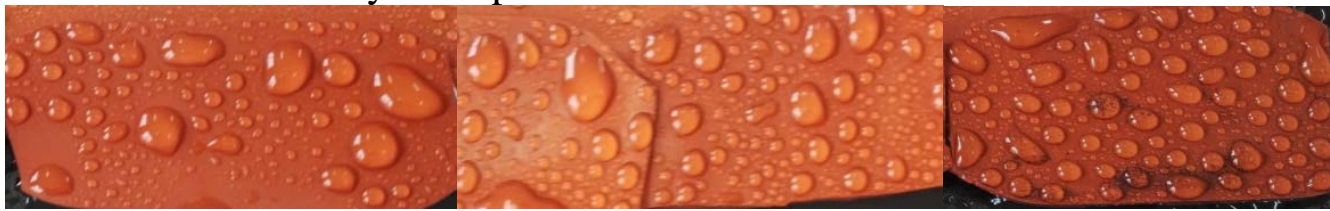
(a) 5 days
(b) 10 days
(c) 20 days

Fig.4 Hydrophobicity classification of samples after cleaning

\section{Influence factors.}

(a)NSDD and ESDD

Equivalent salt deposit density (ESDD) and nonsoluble deposit density(NSDD) were adopted to represent the degree of filth. According to the test result (Fig.5), ESDD was higher than NSDD for five days' contamination, NSDD and ESDD were all rising in time, but growth speed of NSDD was faster than ESDD, and after ten days accumulating dirt, NSDD was finally greater than ESDD, and then continue above ESDD.

Combined with filth degree and hydrophobic result, when contaminated more than 10 days, and ESDD reached $0.13 \mathrm{mg} / \mathrm{cm}^{2}$, and NSDD reached $0.16 \mathrm{mg} / \mathrm{cm}^{2}$, hydrophobicity were greatly weakened. These could be considered as one of the impact parameters and boundary conditions.

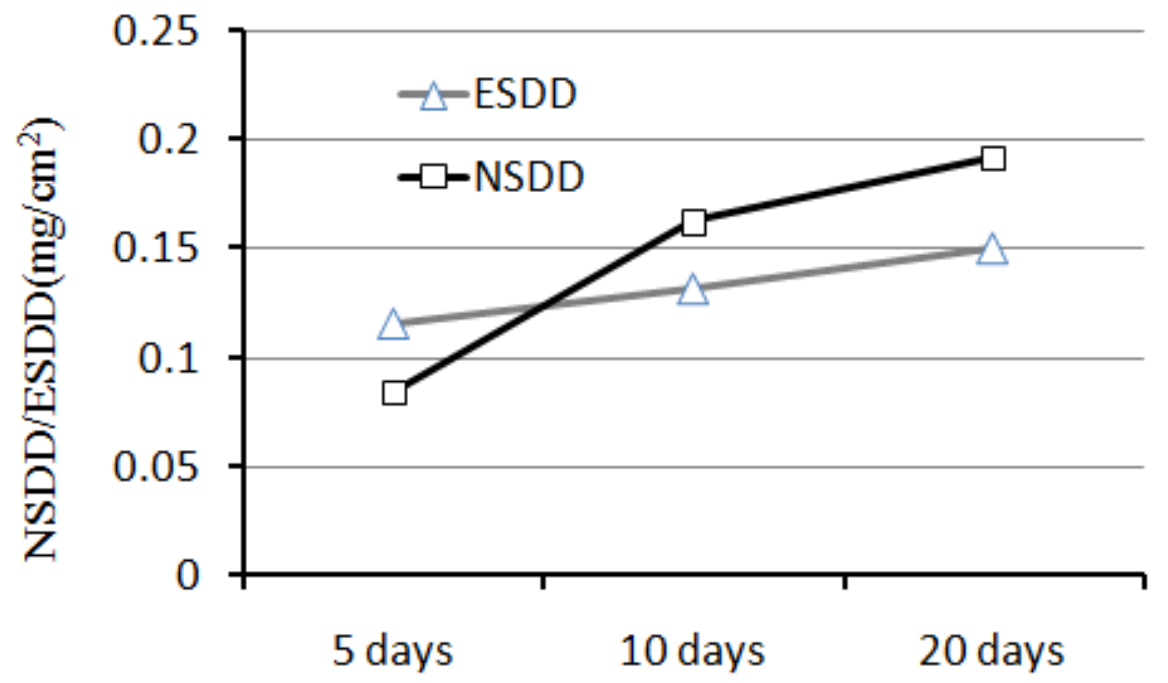

Fig.5. Equivalent salt deposit density(ESDD) and nonsoluble deposit density(NSDD) test of fouling samples 
(b) Temperature

After the polluted samples were heated on the $80^{\circ} \mathrm{C}$ hot stage for eight hours, the hydrophobicity was greatly improved (Fig.6). For samples of ten days contamination, the hydrophobicity could rise from HC5 to HC3-HC4, for samples of twenty days contamination, the surface emerged certain dry area, and didn't be wetted completely as before. Thus it can be seen that, temperature rising could accelerate the molecular activity, low molecular weight material and chain segment would become active, and could move up to dirt on surface more easily, this course made the surface more hydrophobic. On the contrary, when temperature fall down to a certain degree, migration could become very hard, this is unfavorable to restore hydrophobic surface after accumulated dirt to a certain extent.

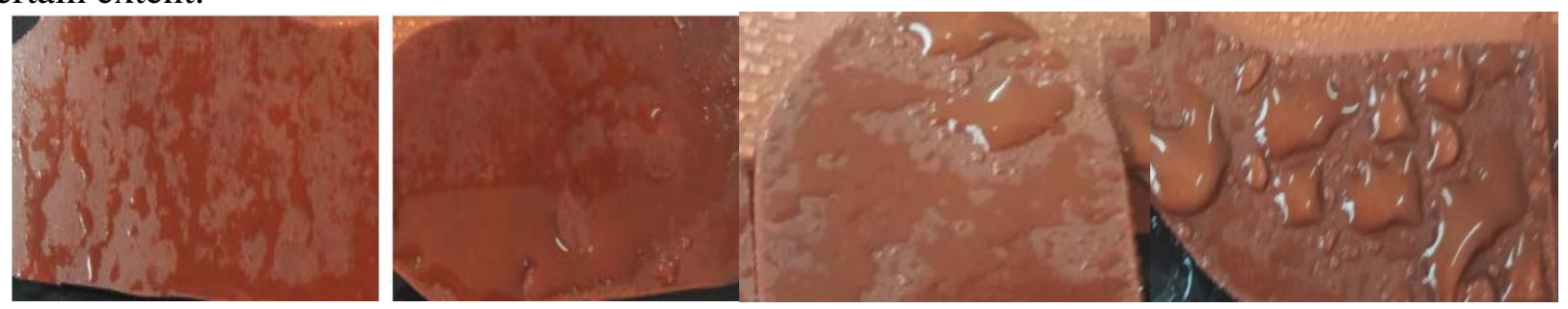

(a) Ten days of contamination(before heating)

(b) Ten days of contamination(after heating)

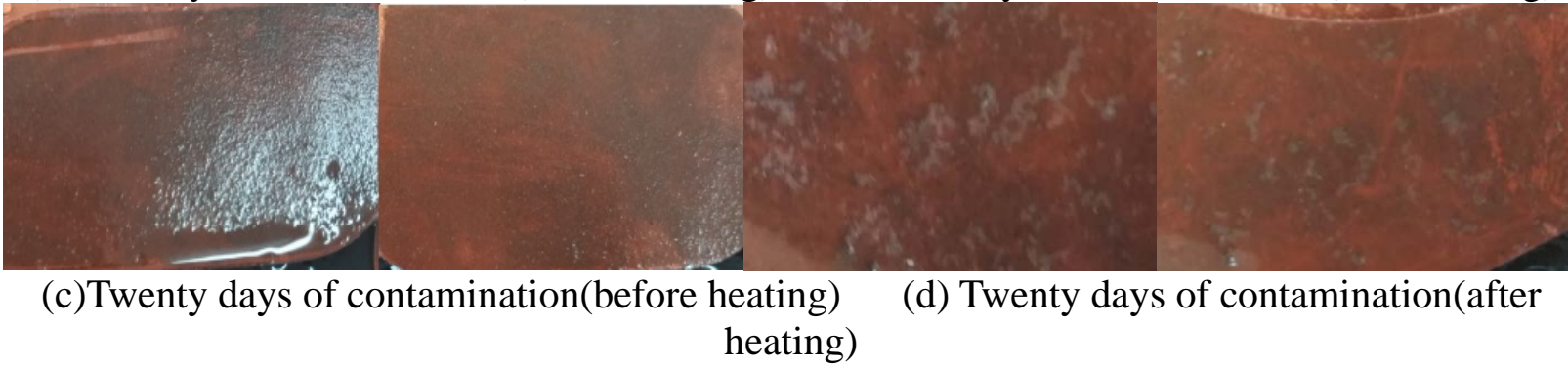

Fig.6. Effect of temperature on the transfer of hydrophobicity

\section{Conclusion}

Silicone rubber samples were placed under simulated winter fog-haze chamber to accumulating pollution, It's found that, the longer contamination time was, the worse the transfer of hydrophobicity was. The reason was related to the pollution degree and surrounding temperature. When NSDD and ESDD reach a certain degree, for example ESDD was $0.13 \mathrm{mg} / \mathrm{cm}^{2}$, NSDD was $0.16 \mathrm{mg} / \mathrm{cm}^{2}$ in this paper, hydrophobicity and transfer of hydrophobicity would become very poor. Low temperature is another one that could hinder the hydrophobic transfer of molecular or chain segment on to the dirty surface.

\section{Acknowledgement}

In this paper, the research was sponsored by the State Grid Science Foundation (Project No. 521700140004) and Guodian Futong Corporation Fund of Science and Technology Project (Project No. FTZY201601).

\section{References}

[1] Qin Rui, Sun Zhe, Wang Ying. Study on RTV Anti-Contamination Flashover Coatings[J].Modern paint \&Finishing, 2009,12(9), 4-7, 10.

[2] Li Jin-hui, Shao Hui-mei, Zhang Min, etal. Progress in anti-pollution-flashover temperature vulcanized silicone rubber coating[J], New Chemical Materials, 2012, 40(1),25-27.

[3]Li Peng, Ma Bin, Liu Dao-hui, etal. Effect of Ultraviolet radiation aging on the hydrophobicity of composite silicone rubber insulators[J], Electric Power, 2015, 48(3),80-83,98. 
[4] He Bo, Wan Jun, Zhang Gang, etal. Study on Hydrophobicity and Withstand Characteristics of Natural Aging Silicone Rubber[J], High Voltage Apparatus, 2009, 45(5), 16-19.

[5] Wang Xiu-juan, Wang Gang, Xu Ao-ran, etal. Study of DC Arc Characteristics on Polluted Silicon Rubber Surface[J], Insulators and Surge Arresters, 2015, (6), 34-38.

[6] ZHAO Ying. Influence of Fog-Haze to Hydrophobic of Composite Insulator[J], Insulators and Surge Arresters, 2015, (1), 45-48,53.

[7] Xu Zhi-niu, Lv Fang-cheng, Li Man, etal. Influence of Temperature on Silicone Rubber Hydrophobicity Caused by Corona[J], High Voltage Engineering, 2011, 37(1), 69-76.

[8] Liu Yang, Hou Zhi-cheng, Wei Xu, etal. Effect of Environment Factor on Hydrophobicity andTransfer of Hydrophobicity of Silicone Rubber[J], High Voltage Engineering, 2010, 36(10), 2454-2459.

[9] Song Yi, Analysis on Saturated Wetting Characteristic of Pollution Layer on Insulator in Cold Fog Environment[J], High Voltage Apparatus, 2014, 50(11), 122-127.

[10] SU Zhiyi. Influences of Fog-Haze on External Insulation of Transmission and Distribution Equipments[J], Power System Technology, 2013, 37(8), 2284-2290. 